\title{
Biometría del Músculo Grácil: Pedículos Vasculares e Inervación en un Grupo de Individuos Brasileños
}

\author{
Biometric Characteristics of the Gracilis Muscle: Vascular Pedicles \\ and Innervation in a Group of Brazilian Individuals
}

\author{
San Martín, N. ${ }^{1,2}$; Sousa-Rodrigues, C. F. ${ }^{3} \&$ Olave, E. ${ }^{4}$
}

\begin{abstract}
SAN MARTíN, N.; SOUSA-RODRIGUES, C. F. \& OLAVE, E. Biometría del músculo grácil: pedículos vasculares e inervación en un grupo de individuos brasileños. Int. J. Morphol., 38(3):536-544, 2020.

RESUMEN: El músculo grácil (MG) está ubicado en la cara medial del muslo, medial y posterior al aductor largo en su parte proximal. Se origina a nivel del pubis y se inserta en la cara medial de la tibia, en su parte superior. Como colgajo libre funcional ha sido uno de los injertos más utilizados en reconstrucciones diversas, tales como pene, perineo, vagina, pierna, plexo braquial, parálisis facial, lesiones rectales, entre otras. Basado en lo anterior, el objetivo de este estudio fue complementar la anatomía del MG tanto en sus dimensiones como en sus pedículos vasculares e inervación, estableciendo las relaciones biométricas existentes, contribuyendo a la anatomía quirúrgica, en su uso como injerto. Para ello, se utilizaron 30 miembros inferiores de 20 cadáveres de individuos adultos, brasileños, de sexo masculino, 14 derechos y 16 izquierdos; 17 fijados en formol y 13 en glicerina. Se dividió al muslo en 4 cuartiles enumerados de proximal a distal como C1,C2,C3 y C4. Se contabilizó el número de pedículos y se nombraron como pedículo principal (PP), pedículo menor 1 (Pm1), pedículo menor 2 (Pm2) y pedículo menor 3 (Pm3). La longitud media del GM fue de $42,25 \mathrm{~cm} \pm 2,35$ $\mathrm{cm}$ y su ancho promedio de $32,90 \pm 4,86 \mathrm{~mm}$. Con respecto a los pedículos vasculares se encontró un pedículo en 10/30 casos (33,3\%); un pedículo principal y uno menor en 10/30 (33,3\%); un pedículo principal y dos menores en $8 / 30$ (26,7\%) y un pedículo principal y tres menores en $2 / 30$ (6,7 \%). Su inervación siempre procedió del ramo anterior del nervio obturador (RaNO). El punto motor se encontró a una distancia promedio de 7,94 mm proximal al ingreso del pedículo principal en el MG. Los registros biométricos están expresados en tablas. Los resultados obtenidos aportarán al conocimiento anatómico, pudiendo ser utilizados como soporte morfológico a los procedimientos quirúrgicos que involucren al músculo grácil.
\end{abstract}

PALABRAS CLAVE : Anatomía; Músculo grácil; Pedículos vasculares; Inervación; Biometría.

\section{INTRODUCCIÓN}

En los años 70, el colgajo del músculo grácil (CMG) ha sido utilizado para reconstrucción de pene (Orticochea, 1972), reconstrucción perineal (Bartholdson \& Hultén, 1975) y también en reconstrucción vaginal (McCraw et al., 1976).

Posteriormente, el CMG ha sido uno de los injertos más utilizados, no sólo en uso local sino que se ha constituído en un importante colgajo libre funcional (Alfonso \& Cardona, 2013), diversificando su uso, en reconstrucción en la pierna (Conejero et al., 2013), plexo braquial (Bonilla et al., 2013), parálisis facial y parálisis braquial obstétrica (Wei \& Mardini, 2009), reconstrucción perineal por cáncer de vulva (Luna-Merlos et al., 2017), por exenteración pélvica (Chong et al., 2015), lesiones rectales (Yáñez et al., 2015), y fístulas entre el recto y la vagina o uretra (Zmora et al., 2006), entre otras.
Sus ventajas radican en el tamaño, forma, longitud, buena excursión, suministro vascular confiable, un nervio motor de gran longitud, fácil disección, fuerza muscular aceptable, y un mínimo de morbilidad del sitio donante en términos de función y cosmética (Wei \& Mardini), destacando también su efectividad aún después de reparaciones anteriores fallidas (Zmora et al.).

El músculo grácil (MG) es de anatomía constante (Bonilla et al.), teniendo una forma de banda larga, aplanado transversalmente, más ancho por proximal que por distal (Rouvière \& Delmas, 2005) y con un largo tendón distal. Está ubicado en la cara medial del muslo, medial y posterior al aductor largo en su parte proximal. Su origen es amplio y se extiende desde la sínfisis hasta la rama inferior del pubis. En la parte inferior, el músculo se encuentra inmediatamente posterior al músculo sartorio, donde ambos se cruzan de-

\footnotetext{
${ }^{1}$ Facultad de Ciencias de la Salud, Universidad Adventista de Chile, Chillán, Chile.

${ }^{2}$ Programa de Magíster en Ciencias mención Morfología, Universidad de La Frontera, Temuco, Chile.

${ }^{3}$ Universidad Estadual de Ciencias da Saude de Alagoas, Maceió, Brasil.

${ }^{4}$ Facultad de Medicina, Universidad de La Frontera, Temuco, Chile.
} 
trás del cóndilo femoral medial (Wei \& Mardini), insertándose en la parte superior de la cara medial de la tibia.

Presenta un patrón vascular de clase II basado en la clasificación de Mathes y Nahai (Wei \& Mardini), es decir, tiene un pedículo vascular dominante y otros menores; de hecho Testut \& Latarjet (1981) describieron la existencia de 4 pedículos escalonados de proximal a distal.

La inervación motora del MG es proporcionada por el ramo anterior del nervio obturador (Whitaker et al., 2012; Chen et al., 2018).

Aunque existen numerosas publicaciones sobre la anatomía del colgajo, no es igual para la anatomía de los pedículos y existen pocos informes sobre los pedículos menores del músculo (Magden et al., 2010).

Basado en lo anterior, el objetivo de este estudio fue especificar la anatomía del MG tanto en sus dimensiones como en sus pedículos vasculares e inervación y establecer las relaciones biométricas existentes y así, contribuir en el quehacer quirúrgico, en su uso como injerto.

\section{MATERIAL Y MÉTODO}

Se utilizaron 30 miembros inferiores (MMII) pertenecientes a 20 cadáveres de individuos Brasileños, adultos, de sexo masculino, 14 derechos y 16 izquierdos; 17 fijados en formol y 13 en glicerina. De la totalidad de miembros disecados 18 pertenecían a 9 cadáveres, mientras que los 12 restantes fueron miembros desarticulados. Estas muestras fueron disecadas en los laboratorios de Anatomía Humana de la Universidade Estadual de Alagoas, Maceió, Brasil.

Se realizó una disección por planos abordando la cara medial del muslo hasta llegar al MG utilizando material quirúrgico "ad-hoc". Para exponer el pedículo principal y el nervio obturador y disecar minuciosamente el MG (sin removerlo) desde su origen hasta su extremo más distal en la "pata de ganso", fue necesario cortar horizontalmente el músculo aductor largo.

Una vez lograda la disección se esquematizó la llegada de los pedículos vasculares, considerando los siguientes puntos de referencias y criterios (Fig. 1):

- Se dividió al muslo en cuartiles enumerados de proximal a distal como $\mathrm{C} 1, \mathrm{C} 2, \mathrm{C} 3$ y $\mathrm{C} 4$ con líneas horizontales equidistantes (LH1,LH2,LH3 y LH4), considerando como referencia la línea bicondílea femoral equivalente a LH4.
- Se trazó una línea vertical (LV) desde el vértice del pubis al cóndilo medial del fémur (límite distal).

Se midió:

- LMI: Longitud del MI, desde espina iliaca antero superior (EIAS) al maléolo medial de la tibia.

- LMu: Longitud de muslo desde el trocánter mayor al cóndilo lateral del fémur.

- Distancia del tubérculo púbico al cóndilo medial del fémur.

- LTMG: Longitud total del MG desde origen a inserción.

- ATMG: Ancho total del MG.

- LP MMG: Longitud de la porción muscular del MG.

- AP MMG: Ancho de la porción muscular del MG.

- LPTMG: Longitud de la porción tendinosa del MG a nivel del origen (-O) e inserción (-I).

- APTMG: Ancho de la porción tendinosa del MG a nivel del origen (- O) e inserción (- I).

Se contabilizó el número de pedículos y se nombraron como pedículo principal (PP), pedículo menor 1 (Pm1), pedículo menor $2(\mathrm{Pm} 2)$ y pedículo menor $3(\mathrm{Pm} 3)$, tomando como referencia su diámetro externo de mayor a menor independiente del cuartil en el que se encontraran.

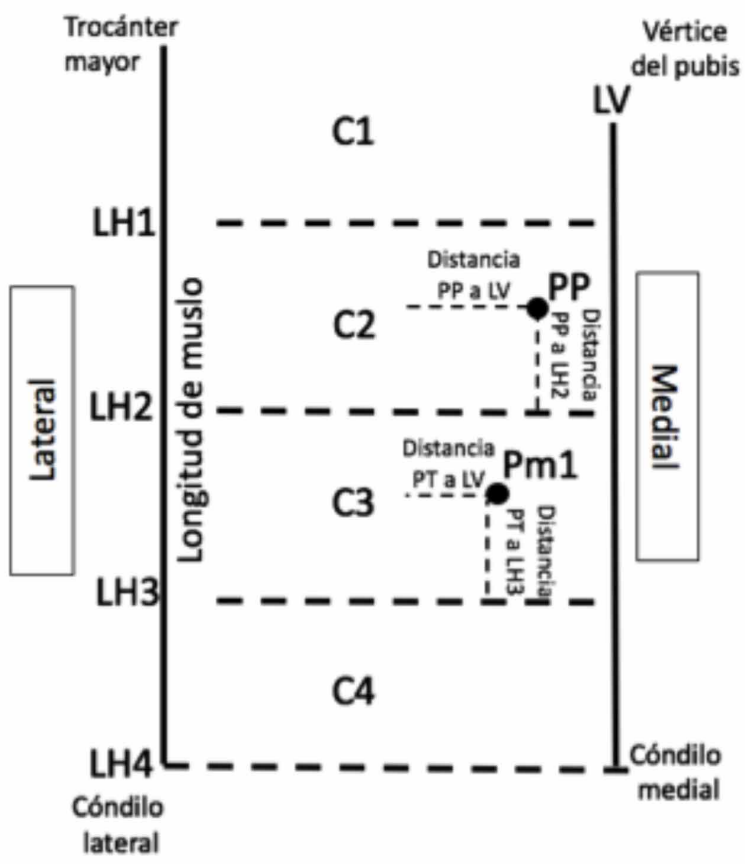

Fig. 1. Esquema que muestra puntos de referencia y mediciones en un miembro inferior derecho.

Relacionado a los pedículos vasculares y ramo nervioso, se midió:

- Longitud de los pedículos.

- Distancia de los pedículos desde su ingreso en el músculo al origen muscular. 
- Ubicación de los pedículos en un determinado cuartil.

- Distancia de los pedículo sobre LH de referencia.

- Distancia de los pedículo respecto a LV (en positivo si el pedículo queda a la derecha y negativo si es a la izquierda).

- Diámetro externo de arteria y venas.

- $\mathrm{N}^{\mathrm{o}}$ de ramos nerviosos (RN)

- Ubicación del RN en un determinado cuartil.

- Longitud y diámetro externo del RN.

- Distancia del punto motor respecto al origen muscular.

- Distancia del RN respecto a LH y LV.

- Si presenta o no divisiones y número de divisiones.

Las medidas se realizaron con un caliper digital marca Mitutoyo ® de 0,01 mm de precisión y las fotografías con una cámara Canon T3i y un lente de 18-55 mm.

La investigación se realizó como una metodología de carácter cuantitativa, no experimental, transversal, descriptiva y el análisis estadístico se llevó a cabo con el programa SPSS versión 25 e incluyó pruebas de estadística descriptiva, de normalidad de Shapiro-Wilk; correlación de Pearson y T de Student para variables paramétricas y correlación de Spearman y U de Mann Whitney para las no paramétricas.

\section{RESULTADOS}

Se registraron las variables LMI y LMu, que fueron en promedio de 86,44 $\pm 4,09 \mathrm{~cm}$ y $48,48 \pm 2,97 \mathrm{~cm}$, respectivamente, mientras que la distancia media del tubérculo púbico hasta el cóndilo medial fue de 40,96 $\pm 2,43 \mathrm{~cm}$ (Tabla I).

La LMI tuvo correlación positiva con LMu ( $\mathrm{p}<0,000$ $\mathrm{R}=0,841)$, con LTMG ( $\mathrm{p}<0,000 \mathrm{R}=0,633)$ y con LPMMG $(\mathrm{p}<0,001 \mathrm{R}=0,592)$. Además hubo correlación entre LMu y LTMG ( $\mathrm{p}<0,001 \mathrm{R}=0,594)$, como también con LP MMG $(\mathrm{p}<0,000 \mathrm{R}=0,650)$ y el diámetro de la arteria del PP ( $<<0,015 \mathrm{R}=0,439)$.

Músculo grácil: El MG se encontró en la zona medial del muslo, medial al músculo aductor largo y corto. Su longitud media fue de $42,25 \mathrm{~cm} \pm 2,35 \mathrm{~cm}$ y su ancho promedio de $32,90 \pm 4,86 \mathrm{~mm}$. Presentaba una zona media muscular y dos extremos tendinosos, cuyas dimensiones de longitud y ancho se encuentran resumidas en la Tabla I.

La LTMG tuvo correlación con la LP MMG ( $\mathrm{p}<0,000$ $\mathrm{R}=0,724)$ y con el APTMG-I ( $<<0,015 \mathrm{R}=0,442)$, no así con el APTMG-O. Asimismo el ATMG se correlacionó con el APTMG-O $(\mathrm{p}<0,000 \mathrm{R}=0,620)$ y la $\mathrm{LP}$ MMG con el APTMG-I ( $p<0,015$ R=0,441), no así con el APTMG-O.

El AP MMG tuvo correlación con el APTMG-O ( $\mathrm{p}<0,000 \mathrm{R}=0,619)$ y no con el APTMG-I ; mientras que la correlación de LPTMG-I fue positiva con el diámetro de la arteria de $\mathrm{Pm} 1(\mathrm{p}<0,016 \mathrm{R}=0,533)$ y el diámetro de la vena 2 de $\operatorname{Pm} 1$ ( $p<0,006 \mathrm{R}=0,590)$.

La LPTMG-O tuvo correlación con el diámetro de la arteria de $\mathrm{Pm} 2$ ( $\mathrm{p}<0,045 \mathrm{R}=0,643$ ) y la LPTMG-I tuvo correlación con el ancho de la vena 1 de Pm2 ( $<<0,045$ $\mathrm{R}=0,642)$.

Pedículos vasculares: Respecto a la frecuencia de aparición, se encontró sólo un pedículo en 10/30 casos (33,3 $\%)$; un pedículo principal y uno menor en 10/30 (33,3\%); un pedículo principal y dos menores en $8 / 30(26,7 \%)$ y un pedículo principal y tres menores en $2 / 30(6,7 \%)$.

Respecto al PP, se observó en todas las muestras. Se conformó en $28 / 30$ casos $(93,3 \%)$ por una rama de la arteria femoral profunda y en $2 / 30(6,7 \%)$ por una rama de la arteria circunfleja femoral medial; mientras que las venas drenaron siempre en la vena femoral profunda.

Tabla I. Datos biométricos de miembro inferior, muslo y músculo grácil.

\begin{tabular}{lrrrrr} 
& N & MÍNIMO & MÁXIMO & $\begin{array}{r}\text { MEDIA } \\
\text { DESVIACIÓN } \\
\text { ESTÁNDAR }\end{array}$ \\
\hline Longitud de miembro inferior - LMI (cm) & & & 97,8 & 86,44 & 4,09 \\
Longitud de muslo - Lmu (cm) & 30 & 77,9 & $48,69,48$ & 2,97 \\
Longitud total MG - LTMG (cm) & 30 & 43,60 & 54,90 & 42,25 & 2,35 \\
Ancho total MG - ATMG (mm) & 30 & 38,30 & 48,20 & 32,90 & 4,86 \\
Longitud porción muscular MG - LPMMG (cm) & 30 & 20,81 & 39,78 & 29,62 & 3,13 \\
Ancho porción muscular MG - APMMG (mm) & 30 & 23,60 & 34,90 & 32,89 & 4,85 \\
Longitud porción tendinosa MG-origen - LPTMG -O & 30 & 20,81 & 39,78 & 27,41 & 5,65 \\
Longitud porción tendinosa MG-inserción - LPTMG -I & 30 & 18,35 & 44,61 & 154,10 & 42,84 \\
Ancho porción tendinosa MG-origen - APTMG - O (mm) & 30 & 52,79 & 260,00 & 30,24 & 4,33 \\
Ancho porción tendinosa MG-inserción - APTMG -I (mm) & 30 & 19,11 & 42,29 & 3,97 & 0,60 \\
Distancia tubérculo púbis - cóndilo medial (cm) & 30 & 37,00 & 47,00 & 40,96 & 2,43 \\
\hline
\end{tabular}


El PP se encontró en todos los casos en el cuartil 2 (Fig. 2); las distancias promedio respecto a $\mathrm{LV}$ fueron de $1,83 \pm 5,71 \mathrm{~mm}$ y respecto a LH2 de 58,80 $\pm 14,63 \mathrm{~mm}$. Su longitud media fue de 71,99 \pm $12,33 \mathrm{~mm}$. La distancia entre el ingreso en el músculo al origen muscular del MG fue de 90,03 $\pm 12,80 \mathrm{~mm}$ y el promedio del diámetro externo de la arteria fue de 2,66 $\pm 0,51 \mathrm{~mm}$, de la venal de 2,08 \pm $0,66 \mathrm{~mm}$ y de la vena 2 de $1,77 \pm 0,53 \mathrm{~mm}$ (Tabla II).

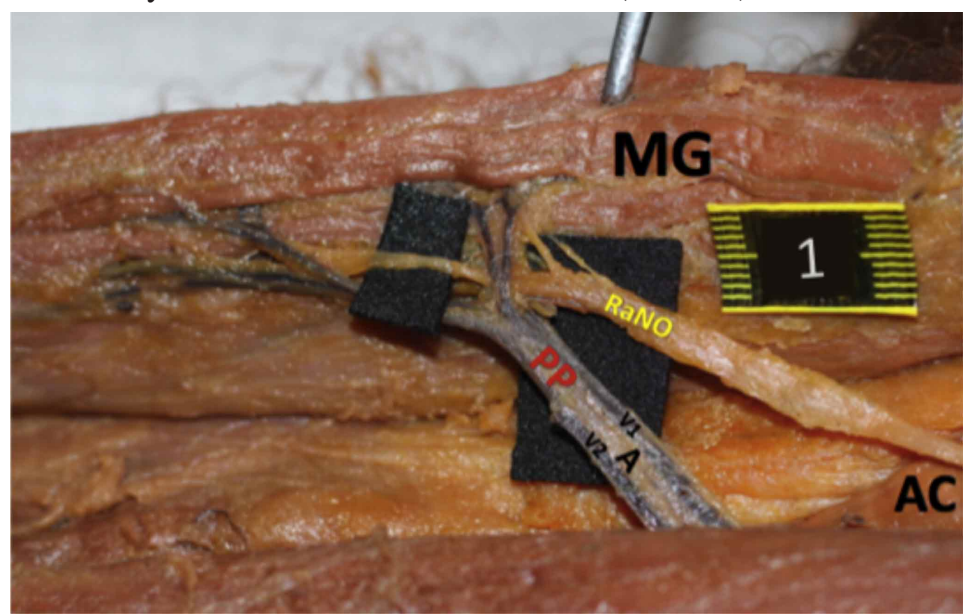

Fig. 2. Entrada de pedículo principal (PP) con su arteria (A) y venas (V1 y V2) y ramo anterior del nervio obturador (RaNO) en el músculo grácil (MG). Se observa cómo ambos terminan en pequeños ramos y ramas entrecruzadas al entrar al MG, y la distancia entre ellos, siendo el RaNO mas proximal.
Existe correlación entre el diámetro de la arteria de PP con el diámetro de su vena 1 ( $\mathrm{p}<0,000 \mathrm{R}=0,602)$ ydiámetro de su vena 2 ( $\mathrm{p}<0,006 \mathrm{R}=0,487)$; como a su vez, también se correlacionan estas dos últimas variables ( $<<0,000 \mathrm{R}=0,817)$.

Con respecto a los demás pedículos, el Pm1 estuvo presente en 20 muestras $(66,7 \%)$. Provino en 20/20 de la arteria femoral superficial. Se encontró en el cuartil 1 en dos casos, en el cuartil 2 en cuatro casos y en el cuartil 3 en catorce casos. La distancia respecto a $\mathrm{LV}$ fue de 0,89 \pm 6,91 mm y tanto en Pm1, Pm2 y Pm3 las distancias sobre LH no se describieron pues los pedículos no siempre estuvieron en el mismo cuartil y por lo tanto no sobre la misma LH.

Con respecto a los pedículos $\mathrm{PP}, \mathrm{Pm} 1$, Pm2 y Pm3 (Fig. 3), los registros de sus variables se encuentran en la Tabla III.

Hubo sólo correlación positiva entre el diámetro de la arteria del Pm1 con el diámetro de su vena $2(p<0,007 R=0,579)$.

Tabla II. Datos biométricos de pedículo principal (PP) y ramo anterior del nervio obturador (RaNO) para el músculo grácil.

\begin{tabular}{|c|c|c|c|c|c|}
\hline & $\mathrm{N}$ & MÍNIMO & MÁXIMO & MEDI A & $\begin{array}{c}\text { DESVIACIÓN } \\
\text { ESTÁNDAR }\end{array}$ \\
\hline PP distancia a origen muscular $(\mathrm{mm})$ & 30 & 67,89 & 112,00 & 90,03 & 12,80 \\
\hline PP distancia proximal a LH2(mm) & 30 & 26,63 & 88,52 & 58,80 & 14,63 \\
\hline PP distancia a LV (mm) & 30 & $-7,65$ & 15,20 & 1,83 & 5,71 \\
\hline PP longitud (mm) & 30 & 49,17 & 96,34 & 71,99 & 12,33 \\
\hline PP diámetro externo arteria $(\mathrm{mm})$ & 30 & 1,68 & 3,54 & 2,66 & 0,51 \\
\hline PP diámetro externo vena 1 (mm) & 30 & 0,88 & 3,28 & 2,08 & 0,66 \\
\hline PP diámetro externo vena $2(\mathrm{~mm})$ & 30 & 0,88 & 3,12 & 1,77 & 0,53 \\
\hline RaNO longitud (mm) & 30 & 74,74 & 139,81 & 96,84 & 14,72 \\
\hline RaNO diámetro externo $(\mathrm{mm})$ & 30 & 1,94 & 5,19 & 3,07 & 0,66 \\
\hline RaNO distancia punto motor a origen muscular & 30 & 57,69 & 121,00 & 82,09 & 15,89 \\
\hline RaNO distancia proximal a LH2 $(\mathrm{mm})$ & 30 & 24,24 & 98,10 & 62,32 & 17,03 \\
\hline RaNO distancia a LV (mm) & 30 & $-10,75$ & 11,80 & 1,23 & 5,54 \\
\hline
\end{tabular}

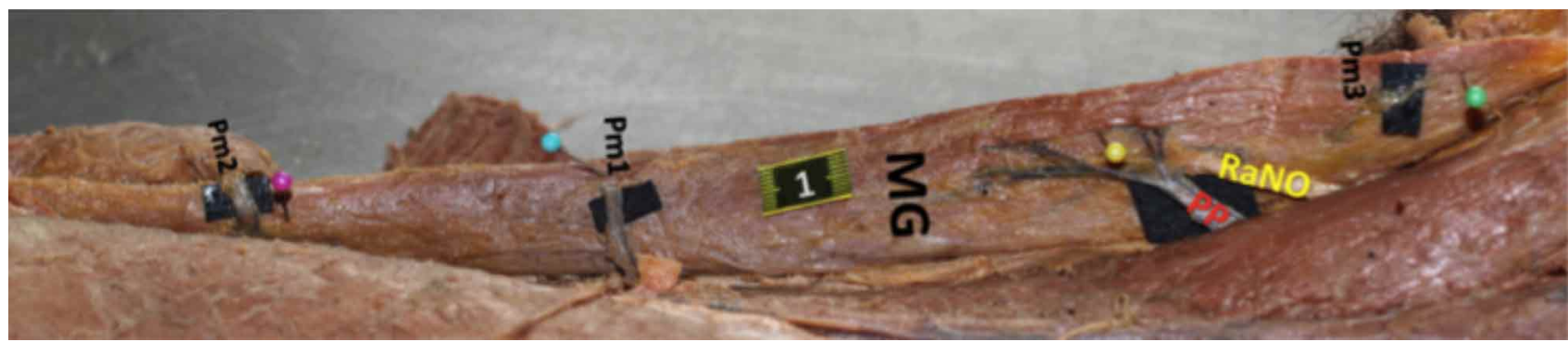

Fig. 3. Músculo grácil (MG) con cuatro pedículos. Pedículo principal (PP), pedículo menor 1 (Pm1), pedículo menor 2 (Pm2) y pedículo menor 3 (Pm3), entrando en la cara profunda del MG. Se observa proximal al PP, el ramo anterior del nervio obturador (RaNO). 
Tabla III. Datos biométricos de los pedículos menores (Pm1, Pm2, Pm3) del músculo grácil.

\begin{tabular}{|c|c|c|c|c|c|}
\hline & $\mathrm{N}$ & MÍNIMO & MÁXIMO & MEDIA & $\begin{array}{c}\text { DESVIACIÓN } \\
\text { ESTÁNDAR }\end{array}$ \\
\hline Pm1 distancia a origen muscular (mm) & 20 & 26,90 & 254,00 & 155,11 & 73,06 \\
\hline Pm1 distancia a LV (mm) & 20 & $-13,65$ & 14,93 & 0,89 & 6,91 \\
\hline Pm1 longitud (mm) & 20 & 24,32 & 106,27 & 54,49 & 18,62 \\
\hline Pm1 diámetro externo arteria (mm) & 20 & 0,72 & 2,73 & 1,73 & 0,54 \\
\hline Pm1 diámetro externo venal (mm) & 20 & 0,73 & 2,52 & 1,24 & 0,42 \\
\hline Pm1 diámetro externo vena2 (mm) & 20 & 0,58 & 2,06 & 1,18 & 0,37 \\
\hline Pm2 distancia a origen muscular (mm) & 10 & 208,00 & 296,00 & 251,62 & 28,09 \\
\hline Pm2 distancia a LV (mm) & 10 & $-34,69$ & 8,03 & $-1,76$ & 11,98 \\
\hline Pm2 longitud (mm) & 10 & 13,90 & 92,82 & 45,66 & 30,32 \\
\hline Pm2 diámetro externo arteria $(\mathrm{mm})$ & 10 & 0,80 & 1,95 & 1,26 & 0,31 \\
\hline Pm2 diámetro externo venal (mm) & 10 & 0,23 & 1,33 & 0,75 & 0,27 \\
\hline Pm2 diámetro externo vena2 (mm) & 10 & 0,53 & 1,20 & 0,78 & 0,19 \\
\hline Pm3 distancia a origen muscular (mm) & 2 & 64,51 & 309,00 & 186,76 & 172,88 \\
\hline Pm3 distancia a LV (mm) & 2 & $-4,47$ & $-3,47$ & $-3,97$ & 0,71 \\
\hline Pm3 longitud (mm) & 2 & 10,42 & 26,92 & 18,67 & 11,67 \\
\hline Pm3 diámetro externo arteria (mm) & 2 & 0,83 & 0,90 & 0,87 & 0,05 \\
\hline Pm3 diámetro externo venal (mm) & 2 & 0,58 & 0,66 & 0,62 & 0,06 \\
\hline Pm3 diámetro externo vena2 (mm) & 2 & 0,64 & 0,66 & 0,65 & 0,01 \\
\hline
\end{tabular}

El Pm2 estuvo presente en 10 muestras. En 9/10 casos la rama provino de la arteria femoral superficial y en 1 caso de la arteria femoral profunda.

Se encontró en un caso en el cuartil 1, siete casos en el cuartil 3 y dos casos en el cuartil 4 .

El diámetro de la vena 1 con la vena 2 del Pm2 tuvo correlación ( $\mathrm{p}<0,003 \mathrm{R}=0,836$ ).

El Pm3 estuvo presente en 2 muestras y en ambos casos estas provino de la arteria femoral superficial. Se encontró un caso en el cuartil 1 y el otro en el cuartil 4.

Inervación: Siempre fue dada por el RaNO, que pasó a través del foramen obturador y se dirigió oblicuamente de medial a lateral y de proximal a distal. Se relacionó profundamente al músculo pectíneo, entre el músculo aductor largo por anterior y el músculo aductor corto por posterior (Fig. 4). Ingresó al músculo en el $100 \%$ de los casos en el cuartil 2 por su cara profunda, a una distancia media desde el origen muscular de $82,09 \mathrm{~mm}$ $\pm 15,89 \mathrm{~mm}$.

El ramo anterior del nervio obturador (RaNO) llegó directo al MG en 12/30 casos (40 \%) y emitió ramos secundarios (RS) en 18/30 casos (60\%). En 7/18 (38\%) emitió un RS; en 10/18 (56 \%) dos RS y en 1/18 (6\%) tres RS. Cuando no emitió RS, el punto motor del RaNO, se encontró a una distancia promedio de 7,94 mm proximal al ingreso del PP en el MG. Los registros biométricos del RaNO están en la Tabla II.

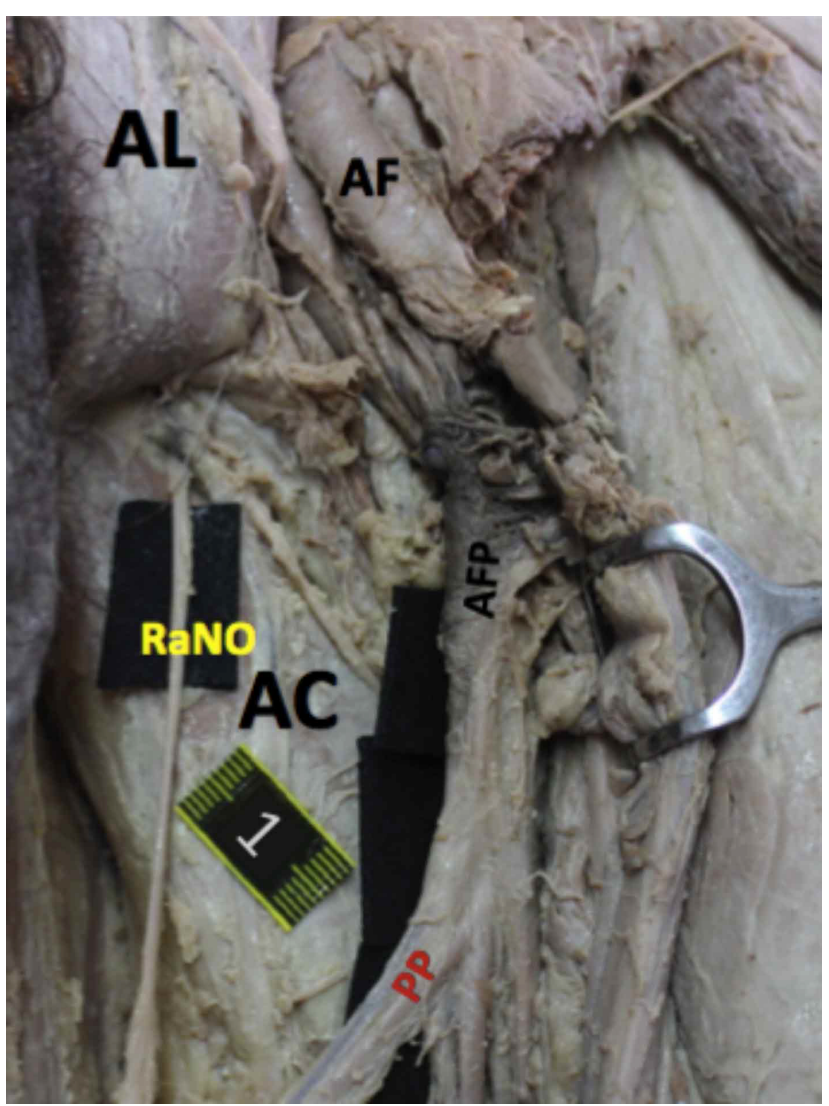

Fig. 4. Músculo aductor largo (AL) seccionado y reclinado, que permite exponer al ramo anterior del nervio obturador (RaNO). RaNO descansa sobre el músculo aductor corto (AC). Se observa pedículo principal (PP) que proviene de la arteria femoral profunda (AFP) y ésta a su vez, de la arteria femoral (AF). 
Según la prueba de Spearman, no hubo correlaciones entre las variables descritas y el $\mathrm{n}^{\circ}$ de pedículos presentes, así como tampoco existió diferencias estadísticas significativas entre muslos derecho e izquierdo $(p>0,05)$ de acuerdo a la prueba $U$ de Mann Whitney en variables no paramétricas, ni tampoco en las variables paramétricas separadas por grupo derecho e izquierdo según la prueba T- Student $(p>0,05)$.

\section{DISCUSIÓN}

Respecto a la LMI, Bonilla et al., en su estudio en población Argentina encontraron una media de 79,82 \pm $67,15 \mathrm{~cm}$, mientras que en nuestro estudio en población brasileña la LMI fue superior $(86,44 \pm 4,09 \mathrm{~cm})$. Asimismo la distancia media del tubérculo púbico hasta el cóndilo medial fue de 40,96 $\pm 2,43 \mathrm{~cm}$, superior a la que encontró Kwon et al. (2009) con una media de 37,7 $\pm 2 \mathrm{~cm}$.

Existe bastante discrepancia respecto a la longitud del MG, Wei \& Mardini encontraron un rango de 20 a $30 \mathrm{~cm}$, similar a lo que describe Conejero et al. (30 $\mathrm{cm})$; nuestro estudio en cambio, reportó una media de $42,25 \mathrm{~cm}$, más cercano a lo que había descrito Bonilla et al. (432,63 mm) y Dziedzic et al. (2018) con una media de $482 \mathrm{~mm}$.

El MG es alargado y limitado por dos tendones, con fibras musculares fusiformes que en todos los casos se confundieron en su extremo distal con un largo y angosto tendón de inserción. Dicho tendón tenía entonces, una porción intra y extramuscular, por lo que no estamos de acuerdo con la afirmación de Davis et al. (2012) respecto a que el MG no tendría tendón intramuscular.

Respecto al ancho del MG la media encontrada fue de 32,90 mm, menor a lo que describe Bonilla et al. $(40,06$ $\mathrm{mm})$ y Conejero et al. $(40-60 \mathrm{~mm})$.

Bonilla et al. con respecto a la longitud de la P MMG reportaron una media de $31,30 \mathrm{~cm}$, levemente mayor a los encontrados en este estudio.

La longitud del tendón en su extremo distal fue de $154,10 \mathrm{~mm}$, levemente superior a lo encontrado por Wei \& Mardini (150 cm o más). En la literatura referenciada no se entrega información respecto a la longitud del tendón en su extremo proximal, ni al ancho del tendón en los extremos; sin embargo, en nuestro estudio los valores promedio encontrados fueron $27,41 \mathrm{~mm} ; 30,24 \mathrm{~mm}$ y 3,97 $\mathrm{mm}$, respectivamente (Tabla I).
En esta investigación se observó que el PP estaba conformado por la arteria femoral profunda $(93,3 \%)$, similar a lo que describen Whitaker et al. $(92,3 \%)$ y Magden et al. $(86,7 \%)$ y diferente a lo que describen otros autores que Magden et al. citaron en el artículo referenciado. En este aspecto, existe discrepancia aún sobre el origen arterial del PP. En el resto de los casos $(6,7 \%)$ la irrigación fue dada por la arteria circunfleja femoral medial, que es la otra arteria que se nombra en las descripciones anatómicas. En todos los casos el drenaje fue en la vena femoral profunda, igual que lo describe Wei \& Mardini y Bonilla et al.; no obstante, no hay registro del drenaje de los pedículos menores. En nuestro estudio, también drenaban en la vena femoral profunda.

La distancia en que el PP ingresó al MG respecto a su origen muscular $(90,03 \mathrm{~mm})$ es inferior a lo que describió Magden et al. (148 $\mathrm{mm}$ ) y hay varios autores que establecieron un margen bastante amplio, refiriéndose además en unidad de centímetros; por ejemplo Wei \& Mardini (6$12 \mathrm{~cm})$, Whitaker et al. $(8-12 \mathrm{~cm})$ y Bonilla et al. $(8-10$ $\mathrm{cm}$ ), no obstante, siendo rangos semejantes con nuestro estudio.

Asimismo en la longitud del PP los autores Magden et al. y Bonilla et al., tuvieron una media de 54 y 39,71 $\mathrm{mm}$ respectivamente; menores a lo que encontramos en nuestro estudio $(71,99 \mathrm{~mm})$.

Concordamos con Wei \& Mardini en cuanto a que, una buena disección del PP y del RaNO, requieren una excursión retrógrada, es decir, disecar hacia proximal hasta llegar al foramen obturador, lo que alarga de $2-4 \mathrm{~cm}$ las disecciones y las acerca al valor real. En nuestro trabajo, en todos los casos, hicimos un corte horizontal en el músculo aductor largo para la exposición de lo antes mencionado.

El diámetro externo de la arteria y venas fue similar a lo reportado por Wei \& Mardini $(1,5 / 2 \mathrm{~mm})$ y Bonilla et al. $(1,17 / 1,9 \mathrm{~mm})$.

Hay bastante discrepancia o poca claridad tanto en la terminología de los pedículos, como en los criterios para su clasificación como menores o accesorios. En base a la revisión de la literatura, decidimos llamarle Pedículo Principal y Pedículos Menores teniendo como criterio el diámetro de los pedículos (Wei \& Mardini; Magden et al.; Whitaker et al.; Conejero et al.; An et al., 2016).

En base a la falta de concenso, no consideramos beneficioso el hacer comparación de nuestros registros de los pedículos menores respecto a otros estudios, sino que 
los dejamos a disposición del lector, sugiriendo en otras investigaciones utilizar nuestro criterio de selección.

Respecto a la frecuencia de aparición de los pedículos existe bastante discrepancia entre los autores y lo informado en esta investigación. Se encontró 10/30 casos $(33,3 \%)$ con un solo pedículo el cual correspondía al principal, mientras que Whitaker et al. reportó 2/28 casos $(7,1 \%)$ y Bonilla et al. 5/28 (18,7\%).

Hubo también 10/30 casos $(33,3 \%)$ en que se encontró un pedículo principal y uno menor, a diferencia de An et al. que informó 14/28 casos (50\%), Whitaker et al. 7/27 (25,9\%) y Bonilla et al. 12/28 (43,7\%).

Se encontró un pedículo principal y dos accesorios en 8/30 casos (26,7 \%), similar a lo reportado por An et al. $(28,6 \%)$ y menor a lo que describe Whitaker et al. $(51,9 \%)$ y Bonilla et al. $(37,5 \%)$.

Finalmente hubo $2 / 30$ casos $(6,7 \%)$ con un pedículo principal y 3 accesorios (Fig. 4), mientras que An et al. y Whitaker et al. reportaron 4/28 (14,3\%) y 6/ $27(22,2 \%)$ respectivamente; siendo en los tres estudios la situación menos común.

Textos clásicos de anatomía y todos los artículos referenciados señalan que el MG está inervado por el RaNO, esto también fue evidenciado en todos los casos.

La distancia del punto motor respecto al origen muscular que observamos $(82,09 \mathrm{~mm})$ es menor que lo que describen Magden et al. $(94,4 \mathrm{~mm})$ y Bonilla et al. (12,94 mm), así como también encontramos que el punto motor estaba a una media de $7,94 \mathrm{~mm}$ por sobre el ingreso del PP; siendo en todos los casos proximal a éste; a diferencia, Wei \& Mardini refieren una distancia de 1-2 $\mathrm{cm}$ y Bonilla et al. 2-3 cm.

Referente a la longitud del RaNO, fue necesario cortar el aductor largo para exponer bien su origen en la bifurcación y la longitud evaluada $(96,84 \mathrm{~mm})$ es muy similar a lo estudiado por Bonilla et al. $(95,81 \mathrm{~mm})$; así como el diámetro externo (Tabla II). No hay mayores antecedentes respecto a estos detalles anatómicos; la literatura clásica no entrega ningún detalle biométrico y los artículos revisados, a veces, dejan un margen muy amplio; lo que es muy relevante, pues conocer tanto la longitud del pedículo como la del único ramo nervioso es primordial para la viabilidad del colgajo y su anclaje (Bonilla et al.).

Respecto a la cantidad de ramos nerviosos sólo
Bonilla et al. reportaron haber encontrado en un 18,75 $\%$, dos ramos; en nuestro caso al igual que lo describe la literatura clásica (Testut \& Latarjet; Rouvière \& Delmas) encontramos solo uno.

$\mathrm{Al}$ alcanzar el MG, en un $60 \%$ de los casos, el RaNO se dividió hasta en 3 pequeños ramos secundarios justo antes de entrar a la fibra muscular, igual como lo observó An et al. y Chen et al. quienes describieron que se puede subdivir en 2 a 4 ramos secundarios. Estos últimos, también describieron que por debajo de estos pequeños ramos nerviosos, existen pequeños vasos sanguíneos entrando al músculo, situación que también observamos, donde se entremezclaban los pequeños ramos nerviosos y las ramas del PP.

Esta situación fue particularmente dificultosa para la disección y medición; por lo que a fin de evitar daños en las muestras, se consideró como referencia para mediciones, el punto motor y la rama arterial más gruesa. Consideramos que este antecedente no tendría tanta relevancia, no obstante, podría abrir paso a una nueva investigación.

Respecto a estudio de correlaciones, sólo Bonilla et al. presentaron antecedentes para contrastar nuestros hallazgos. Hubo correlación entre LMI, LMu, LTMG y LP MMG, no obstante no hubo correlaciones entre las anteriores mencionadas y ATMG, AP MMG ni LPTMG (tanto origen como inserción), no así Bonilla et al. quienes si obtuvieron correlación de la LMI con el LPTMG y con su ancho.

Sin embargo, si hubo correlación entre el LTMG y el LP MMG, así como el APTMG; también entre el ATMG y el AP MMG y APTMG y estas dos entre sí también. Todo lo anterior establece una relación proporcional que se podría explicar en base a la funcionalidad de un músculo.

Referente al PP, las correlaciones positivas encontradas fueron entre el diámetro externo de la arteria y de las venas igual que Bonilla et al., así como sus venas entre sí; esta correlación entre arteria y vena se repitió también en los pedículos menores.

Bonilla et al. reportaron asociación positiva entre la distancia del PP y el RN en el músculo a su inserción muscular con longitud de miembro inferior, no obstante, en nuestro estudio dichas variables se correlacionaron no con LMI sino con longitud total de MG. De este modo se puede inferir que existe una relación directamente proporcional entre el largo del músculo y la distancia en que ingresa el RN y el PP. 
No obtuvimos ninguna correlación positiva, igual que Bonilla et al., entre las variables descritas y el $\mathrm{n}^{\circ} \mathrm{de}$ pedículos presentes.

Al comparar las variables por muslo derecho e izquierdo, no hubo diferencias estadísticamente significativas $(\mathrm{p}>0,05)$.

Si bien se han reportado publicaciones que aluden al MG, éstas son mayoritariamente con énfasis en su uso como colgajo y algunas con descripciones anatómicas más generales o que no coinciden tanto entre sí.

Nuestra investigación viene a ser, por lo tanto, un aporte al conocimiento de la anatomía del músculo grácil, su inervación y además sus pedículos, entregando datos biométricos y sobre sus propias relaciones, que pueden ser muy útiles al momento del abordaje quirúrgico, disminuyendo los riesgos de lesión de los pedículos y nervio y asegurando la viabilidad del colgajo.

SAN MARTÍN, N.; SOUSA-RODRIGUES, C. F. \& OLAVE, E. Biometric characteristics of the gracilis muscle: vascular pedicles and innervation in a group of Brazilian individuals. Int. J. Morphol., 38(3):536-544, 2020.

SU MMARY: The gracilis muscle (GM) is located in the medial aspect of the thigh, medial and posterior to the long adductor in its proximal part. It originates at the pubic level and is inserted in the medial face of the tibia, in its upper part. As a functional free flap, it has been one of the most co mmonly used grafts in various reconstructions, such as penis, perineum, vagina, leg, brachial plexus, facial paralysis, rectal lesions, among others. Based on the above, the objective of this study was to complement the anatomy of the GM both in its dimensions and in its vascular pedicles and innervation, establishing the existing biometric relationships, contributing to the surgical anatomy, in its use as a graft. For this, 30 lower limbs of 20 bodies of adult, Brazilian, male, 14 right and 16 left individuals were used; 17 fixed in formaldehyde and 13 in glycerin. The thigh was divided into 4 quartiles listed from proximal to distal such as $\mathrm{C} 1, \mathrm{C} 2, \mathrm{C} 3$ and $\mathrm{C} 4$. The number of pedicles was counted and they were named as principal pedicle (PP), minor pedicle $1(\mathrm{mP} 1)$, minor pedicle $2(\mathrm{mP} 2)$ and minor pedicle 3 (mP3). The average length of the GM was $42.25 \mathrm{~cm}$ $\pm 2.35 \mathrm{~cm}$ and its average width was $32.90 \pm 4.86 \mathrm{~mm}$. With respect to vascular pedicles, a pedicle was found in $10 / 30 \mathrm{ca}-$ ses (33.3\%); one PP and one $\mathrm{mP}$ in 10/30 (33.3\%); one PP and two $\mathrm{mP}$ in $8 / 30(26.7 \%)$ and one PP and three $\mathrm{mP}$ in $2 / 30$ $(6.7 \%)$. Its innervation always came from the anterior branch of the obturator nerve $(\mathrm{aBON})$. The motor point was found at an average distance of $7.94 \mathrm{~mm}$ proximal to the entry of the PP in the GM. Biometric records are expressed in tables. The results obtained will contribute to anatomical knowledge, and can be used as morphological support for surgical procedures that involve the GM.

KEY WORDS: Anatomy;Gracilis muscle; Vascular pedicles; Innervation; Biometry.

\section{REFERENCIAS BIBLIOGRÁFICAS}

Alfonso, A. \& Cardona, M. Reconstrucción de gran defecto perineal con colgajos musculocutáneos de grácilis. A propósito de un caso. Rev. Colomb. Cancerol., 17(1):29-32, 2013.

An, X.; Dong, P.; Qu, X. \& Li, S. Demonstration of intramuscular artery and nerve distribution in the same whole mount gracilis muscle by different colors. Int. J. Morphol., 34(3):1034-8, 2016.

Bartholdson, L. \& Hultén, L. Repair of persistent perineal sinuses by means of a pedicle flap of musculus gracilis. Case report. Scand. J. Plast. Reconstr. Surg., 9(1):74-6, 1975.

Bonilla, G.; Dodaro, F.; Villamil, F.; Albarracin Reinoso, M.; Rodriguez, M. \& Moyano, S. Músculo gracil: bases de la refuncionalización del plexo braquial. Rev. Argent. Anat. Online, 4(3):85-91, 2013.

Chen, Y.; Wang, M.; Zhang, X.; Zhang, T. \& Yang, S. Localization of the nerve entry points of thighadductor muscles by spiral computed tomography: a novel anatomical guide for chemical neurolysis in treating muscle spasticity. Int. J. Morphol., 36(3):871-6, 2018

Chong, T. W.; Balch, G. C.; Kehoe, S. M.; Margulis, V.\& SaintCyr, M. Reconstruction of large perineal and pelvic wounds using gracilis muscle flaps. Ann. Surg. Oncol., 22(11):373844, 2015.

Conejero, A.; Dagnino, B. \& Pereira, N. Experiencia en reconstrucción de tercio distal de pierna con colgajo libre de músculo gracilis. Cir. Plást. Iberolatinoam., 39(3):279-83, 2013.

Davis, J. A.; Stringer, M. D. \& Woodley, S. J. New insights into the proximal tendons of adductor longus, adductor brevis and gracilis. Br. J. Sports Med., 46(12):871-6, 2012.

Dziedzic, D. W.; Bogacka, U.; Komarnit,ki, I. \& Ciszek, B. Anatomy and morphometry of the distal gracilis muscle tendon in adults and foetuses. Folia Morphol. (Warsz.), 77(1):13843, 2018.

Kwon, J. Y.; Kim, J. S. \& Lee, W. I. Anatomic localization of motor points of hip adductors. Am. J. Phys. Med. Rehabil., 88(4):336-41, 2009.

Luna-Merlos, P.; Salazar-Campos, J. E.; Meneses-García, A. \& Montalvo-Esquivel, G. Reconstrucción perineal con doble colgajo de gracilis en cáncer de vulva. Reporte de caso y revisión de la literatura. Gac. Mex. Oncol., 16(5):299-302, 2017.

Magden, O.; Tayfur, V.; Edizer, M. \& Atabey, A. Anatomy of gracilis muscle flap. J. Craniofac. Surg., 21(6):1948-50, 2010.

McCraw, J. B.; Massey, F. M.; Shanklin, K. D. \& Horton, C. E. Vaginal reconstruction with gracilis myocutaneous flaps. Plast. Reconstr. Surg., 58(2):176-83, 1976.

Orticochea, M. A new method of total reconstruction of the penis. Br. J. Plast. Surg., 25(4):347-66, 1972.

Rouvière, H. \& Delmas, A. Anatomía Humana: Descriptiva, Topográfica y Funcional. Vol. 3. 11 a ed. Barcelona, Masson, 2005. 
Testut, L. \& Latarjet, A. Tratado de Anatomía Humana. Vol. 1. $9^{\text {a }}$ ed. Barcelona, Salvat, 1981.

Wei, F. C. \& Mardini, S. Flaps and Reconstructive Surgery. Taiwan, Saunders Elsevier, 2009.

Whitaker, I. S.; Karavias, M.; Shayan, R.; le Roux, C. M.; Rozen,W. M.; Corlett, R. J.; Taylor, G. I. \& Ashton, M. W. The gracilis myocutaneous free flap: a quantitative analysis of the fasciocutaneous blood supply and implications for autologous breast reconstruction. Plos ONE, 7(5):e36367, 2012.

Yáñez, M. R.; Torres, E. E.; Maíz, H. C.; Cifuentes, O. I.; Deichler, V. F.; Sanhueza, G. M.; Reyes, R. J.; Kusanovicj, B. R. \& León, G. F. Reconstrucción perineal inmediata en extensa resección abdominoperineal rectal. Rev. Chil. Cir., 67(4):41926, 2015.

Zmora, O.; Tulchinsky, H.; Gur, E.; Goldman, G.; Klausner, J. M. \& Rabau, M. Gracilis muscle transposition for fistulas between the rectum and urethra or vagina. Dis. Colon Rectum, 49(9):1316-21, 2006.
Dirección para correspondencia:

Dr. Enrique Olave

Facultad de Medicina

Universidad de La Frontera

Av. Francisco Salazar 01145

Casilla $54 \mathrm{D}$

Temuco

CHILE

Email: Enrique.olave@ufrontera.cl

Recibido : 25-11-2019

Aceptado: 02-01-2020 